\title{
Civilisations
}

Revue internationale d'anthropologie et de sciences

humaines

$44 \mid 1997$

Les peuples des forêts tropicales

\section{Un style de vie en voie de mutation}

Considérations sur les peuples des forêts denses humides

\section{Serge Bahuchet}

\section{(2) OpenEdition}

12 Journals

Édition électronique

URL : http://journals.openedition.org/civilisations/1592

DOI : 10.4000/civilisations. 1592

ISSN : 2032-0442

Éditeur

Institut de sociologie de l'Université Libre de Bruxelles

\section{Édition imprimée}

Date de publication : 1 janvier 1997

Pagination : 16-31

ISBN : 2-87263-122-4

ISSN : 0009-8140

\section{Référence électronique}

Serge Bahuchet, « Un style de vie en voie de mutation », Civilisations [En ligne], 44 | 1997, mis en ligne le 29 juin 2009, consulté le 01 mai 2019. URL : http://journals.openedition.org/civilisations/1592

DOI : 10.4000/civilisations. 1592

(c) Tous droits réservés 


\section{- UN STYLE DE VIE EN VOEE DE MUTATION: \\ Considérations sur les peuples \\ des forêts denses humides:}

Serge BAHUCHET 
Généralement lorsque les Occidentaux évoquent les habitants des forêts tropicales, ils pensent à quelques peuples mythiques, emblématiques: les Pygmées d'Afrique, les Penan de Bornéo ou les Yanomami d'Amazonie. En réalité, tout autour de l'Équateur, plus de douze millions de personnes vivent dans et de la forêt équatoriale, en Afrique centrale, en Amazonie et en Asie insulaire. Dans leur ensemble, les peuples indigènes des forêts équatoriales ne constituent pas une minorité ; c'est une population très importante qui dépend totalement de la forêt pour sa subsistance.

Source de richesses pour les civilisations du monde tempéré depuis plusieurs siècles, les forêts équatoriales font depuis deux décennies l'objet de toutes les attentions, en ce qui concerne leur développement (c'est-à-dire une utilisation économiquement plus efficace de leurs ressources) ou au contraire leur conservation. Les populations indigènes de ces régions se trouvent donc confrontées, qu'elles le veuillent ou non, à des politiques décidées dans d'autres régions (voire dans d'autres continents) et qui leur sont imposées. Cet article, écrit par un ethnologue, tentera de discuter l'importance de cette irruption de l'économie et des valeurs de l'Occident dans la forêt tropicale et des modifications qui en résultent, pour l'indigène comme pour l'ethnologue dans la pratique de son métier.

Un mot d'éclaircissement sur ce terme «indigène» (II] : commode sans être excellent, il désigne pour nous des populations qui vivent en forêt depuis de nombreuses générations et pour lesquelles le mode de vie tourne autour de l'utilisation de la forêt. Cela les distingue des populations «d'immigrants», c'est-à-dire celles qui viennent des régions périphériques, des savanes ou de zones plus ouvertes et qui sont installées sur les terres forestières depuis une ou deux générations seulement.

\section{DIVERSTIÉ DES POPULATIONS FORESTIËRES}

Ces populations qui vivent dans la forêt depuis plusieurs générations représentent environ $4 \%$ de la population totale des pays forestiers. Mais ces douze millions de personnes représentent aussi une immense diversité ethnique et culturelle. On a en effet pu recenser de l'ordre de 1.500 ethnies, groupes ethniques ou langues différentes.

\begin{tabular}{|c|c|c|c|c|}
\hline Régions forestières & Population totale & Populations forestières & $\%$ & $\begin{array}{l}\text { Nombre d'elhnies } \\
\text { forestières indigènes }\end{array}$ \\
\hline Afrique centrale & 54000000 & 3000000 & 5,5 & \pm 150 \\
\hline Amazonie* & 30400000 & 700000 & 2,3 & 234 \\
\hline Philippines & 62400000 & 1600000 & 2,6 & 52 \\
\hline Malaisie pénin. & 14600000 & 100000 & 0,9 & 19 \\
\hline Indonésie** & 170700000 & 4800000 & 2,8 & \pm 95 \\
\hline Bornéo & 12500000 & 950000 & 7,6 & 62 \\
\hline Nouvelle-Guinée & 5400000 & 1000000 & 18,5 & 806 \\
\hline TOTAL & 350000000 & 12150000 & 3,5 & \pm 1418 \\
\hline
\end{tabular}


Cela signifie aussi un très grand morcellement des spécificités et caractéristiques culturelles. Corrélativement, ces sociétés sont presque toujours de très petite envergure. Nous avons pu calculer, pour les trois continents étudiés, que $80 \%$ des groupes comptent moins de 5.000 personnes, voire moins de 3.000 personnes pour la moitié d'entre eux. Seulement $25 \%$ de ces groupes ont une taille supérieure à 10.000 ou 20.000 personnes. Il s'agit donc là de toutes petites sociétés, ce qui n'est pas sans poser des problèmes aux agents qui interviennent auprès d'elles pour le développement ou pour des programmes de conservation de la nature.

Dans ce vaste ensemble, les populations mobiles, celles qu'on a l'habitude d'appeler des "chasseurs-cueilleurs», qui vivent (ou vivaient il y a encore peu de temps) sans pratiquer ellesmêmes l'agriculture, mais sans en ignorer les ressources néanmoins, ne représentent qu'à peu près 300.000 personnes, pour l'ensemble du monde équatorial. Les principaux groupes vivent en Afrique centrale, à Bornéo, en Malaisie et aux Philippines. C'est dire que ces petits groupes, l'archétype de la population forestière dans notre imaginaire occidental, ne représentent que 3\% des habitants des forêts. lin définitive, la majorité cdes populations forestières est constituée de populations rurales ; ce sont des paysans qui pratiquent l'agriculture itinérante et qu'on nomme souvent des essarteurs ${ }^{[?]}$.

\section{UNE ÉCONOMIE FORESTIÈRE}

Dans ce contexte, comment pouvons-nous caractériser une économie forestière? En dépit des variations régionales, de grandes constantes apparaissent. En premier lieu, l'homme trouve dans la forêt la terre, c'est-à-dire la base indispensable à son agriculture, mais il y trouve aussi ses ressources alimentaires et en particulier protéiques, toutes les matières premières pour son habitat, pour sa maison et son artisanat, et encore ses médicaments, ses plantes médicinales. Enfin, ce qui n'est pas le moins important de tout, la forêt, pour la totalité des populations, se confond avec le Monde, avec le Cosmos, dans toute la dimension religieuse que cela implique. La forêt de ce fait focalise à la fois tous les attraits et toutes les craintes dans l'imaginaire des populations dont nous parlons ici. Sans exagérer, la forêt représente véritablement l'élément vital pour l'ensemble de ces ethnies.

Trois points très importants caractérisent l'économie forestière : la technique agricole, le système alimentaire et les relations avec le monde extérieur.

\section{L'agriculture sur brûlis}

Dans tous les cas, l'agriculture pratiquée dans les forêts denses humides est une agriculture itinérante, dans la majorité des cas sur brûlis (dans quelques rares cas il n'y a pas brûlis). Chaque année une famille abat des arbres, les laisse sécher puis les brûle pour planter dans les cendres. Lun des facteurs les plus importants est que ces arbres ne sont que coupés, ils ne sont jamais dessouchés. Cela veut dire qu'une fois la récolte effectuée, pendant un an ou deux ans successifs, la parcelle est abandonnée et la forêt y repousse.

L'abattis sans dessouchage permet aux arbres de recroitre sans hiatus important. Après 
une période d'abandon de nombreuses années, la parcelle sera de nouveau abattue et remise en culture. 15 à 17 ans semblent constituer une durée moyenne pour la jachère, mais des périodes plus longues (plus de 20 ans) sont fréquentes; il n'est pas rare non plus que les agriculteurs abandonnent définitivement cette parcelle (on parlera alors d'une friche). Le système de l'agriculture forestière ne fonctionne que parce qu'il y a une jachère longue ; de plus, c'est un système autorégénérant par le fait même de la jachère, qui permet au sol de reconstituer sa fertilité. Cette phrase lue fréquemment dans les joumaux, «les paysans détruisent la forêt équatoriale ; chaque jour 30.000 hectares partent en fumée», constitue une véritable tromperie : en même temps qu'il y a des arbres qui brûlent, il y en a d'autres qui repoussent, et cette phase-là n'est jamais mentionnée. L'agriculture forestière, gérée sans contraintes externes au système, n'est pas une agriculture destructrice.

Toutes les études botaniques et écologiques développées depuis une quinzaine d'années montrent que l'homme a transformé la forêt en la cultivant depuis plusieurs milliers d'années, sur tous les continents. L'agriculture itinérante sur brûlis fail partie de la dy'namique de la forêt et son cycle fait partie de l'histoire des forêts.

Toutes les forêts du monde ont été parcourues par les hommes. Il n'y a pas de forêt vierge. En ce sens, on affirmera que les sociétés forestières, essarteurs et chasseurs-collecteurs confondus, font partie intégrante de l'écosystème forestier, qu'elles ont contribué à façonner au cours des derniers millénaires.

\section{Sustème alimentaire}

le deuxième point d'importance c'est que, dans tous les cas, les produits de l'agriculture ne forment qu'une partie du régime alimentaire, ils sont toujours complétés par les produits de la chasse, de la pêche et de la collecte. En schématisant, on dira que l'agriculture apporte les éléments lourds et les glucides, alors que la forêt fournit les protéines et les éléments qualitatifs de l'alimentation [?].

Toutes les sociétés dont on parle ici obtiennent leurs protéines animales des ressources sauvages. Par contre, dans tous les cas, l'élevage, réduit, remplit principalement des fonctions sociales, et sa participation à la diète est restreinte aux fêtes. Les peuples d'Amazonie pêchent et chassent activement, ceux d'Asie insulaire affichent une prédilection pour la pêche, ceux d'Afrique centrale pour la chasse. La collecte apporte des plantes alimentaires ou condimentaires (feuilles, tuhercules, fruits, noix) mais aussi des insectes et petits animaux divers (larves, escargots, batraciens, etc.), avec une saisonnalité très marquée, ce qui en constitue d'ailleurs l'une des caractéristiques essentielles.

Ce style alimentaire mixte a des conséquences très importantes en ce qui concerne les territoires utilisés. l'agriculture itinérante nécessite, on l'a vu, des surfaces qui sont considérablement plus importantes que celles qui sont défrichées chaque année. En effet, une famille défrichant un hectare par an, avec une rotation agricole de 12 ou 17 ans, aura en fait besoin de 12 ou 17 hectares. Mais les activités de chasse et de collecte sont pratiquées en forêt non défrichée, au-delà des terroirs agricoles. Ainsi, toutes les 
familles utilisent des surfaces qu'on pourrait appeler des aires ou des territoires de parcours, qui correspondent aux lieux oì elles vont périodiquement pêcher, chasser et effectuer des collectes saisonnières.

De ce fait, pour déterminer le territoire d'une société, on ne doit pas seulement prendre en compte les parcelles qui ont été défrichées, c'està-dire qui portent des marques de transformation, ce qui dalns nombre de pays, est le seul critère pour leur reconnaître une propriété du sol. Il y a, à côté des surfaces cultivées, une surface encore plus importante que les gens ont besoin de parcourir pour obtenir les éléments de l'alimentation et de l'artisanat qui sont nécessaires à leur vie.

\section{Le monde extérieur}

À travers le temps, aucune de ces populations forestières n'est restée isolée à l'intérieur de la forêt, coupée du reste du monde ; aucune d'entre elles n'est autarcique, pas même les Pygmées ou les tribus reculées de la Nouvelle-Guinée. Toutes, depuis des millénaires, à des degrés divers, ont été incluses dans des circuits d'échanges, de commerce, de relations interethniques, par des voies complexes quelquefois sur de très longues distances, dans lesquelles les éléments de la forêt sont toujours présents.

De plus, il faut insister sur le fait que diverses catégories économiques coexistent dans une même région, qui donnent même souvent lieu à des associations d'ethnies complémentaires, durables et institutionnalisées. Ainsi, des associations bien connues de chasseurs-collecteurs avec des agriculteurs (cas des Pygméés d'Afrique ou des Penan de Bornéo). Mais c'est aussi le cas d'ethnies spéciali- sées comme les pêcheurs en eau douce : il existe des associations complémentaires entre pêcheurs et agriculteurs tout le long des grands fleuves africains comme le fleuve Zaïre. De même, les grandes sociétés déjà hiérarchisées de la plaine inondable de l'Amazonie vivaient, jusqu'à ce qu'elles soient balayées par la Conquête, en association commerciale avec les populations de terres hautes qui les fournissaient en produits sauvages de la grande forêt.

Ces associations ont permis le développement à travers les siècles des grands réseaux de commerce à longue distance, destinés aux pays développés hors de la zone équatoriale. Ce fut le cas dans le bassin congolais avant l'implantation coloniale européenne (exportation vers l'Turope d'ivoire et de bois rouge à teinture - parmi de nombreux autres produits mineurs), et des réseaux de commerce d'Asie tropicale - rotin, copal et damar, ivoire, bois parfumés - entre les îles et le continent chinois, qui commence au moins au ve siècle et persiste encore de nos jours (le voyageur chinois Tcheou Ta Kouan rapporte en 1296 que les populations des forêts du Cambodge fournissaient au Céleste Empire graines de cardamome, laque et cornes de rhinocéros). Les racines sauvages de salsepareille de l'Amazonie soignaient les syphilitiques d'Europe aux XVIIIe et XIXe siècles....

La forêt équatoriale n'a jamais été un "cocon" dans lequel les gens seraient restés enfermés en dehors de tout. C'est là un point très important. En conséquence, la plupart de ces sociétés sont monétarisées, elles connaissent l'argent et elles utilisent des produits de leur travail, que ce soit agriculture, récolte ou chasse, 
pour les vendre (sous forme brute, viande par exemple, ou transformée, artisanat) et en obtenir des biens.

\section{REPAÉSENTATIONS SYMPOLLOUUES DE LA FOHÊT}

I)ans le cadre de la définition d' une stratégie globale de conservation de la forêt équatoriale, une prise en compte de l'importance que revêt la forêt pour les populations qui y vivent, et surtout de la représentation qu'elles s'en font, parait primordiale, afin de proposer des mesures susceptibles de recueillir leur soutien et leur participation. Au-delà de la valeur utilitaire, on doit donc s'interroger sur les fonctions symboliques et religieuses attribuées aux arbres, sur les valeurs symboliques positives et négatives accordées à la forêt elle-même par ses habitants, afin de déterminer les éventuels points d'appui de programmes et d'actions de protection et de gestion durable. On peut relever les trois caractères constants suivants $^{[4]}$ :

\section{L'homme organise son milied}

Les membres d'une population donnée délimitent leur écosystème forestier selon diverses composantes, en particulier en reconnaissant et dénommant les catégories de végétation et de paysage. L'espace est organisé d'une façon précise ; certains types d'activités sont toujours effectués dans un paysage particulier, selon les saisons. Il y a partout une différence entre la zone habitée (le village), la zone transformée par les activités agricoles (champs et jachères) et la zone "vierge», la forêt proprement dite, où s'intercalent les forêts de terre ferme, les furêts marécageuses et ripicoles, les forêts de montagne.
Par exemple, trois ethnies du sud-Cameroun, vivant côte à côte, découpent différemment le même milieu naturel. Ies pêcheurs Yasa opposent la mer à la terre ferme et, dans chaque cas, ce qui est loin de ce qui est proche : la "grande forêt" njinga et la «brousse» eici, le "large" munja et la "côte» manga. Le village, où les jardins sont disposés derrière les maisons, est entouré d'un large cercle de champs et de friches. Les essarteurs Mvae mettent l'accent sur la uforêt vierge» (c' est-à-dire jamais cultivée de mémoire d'homme) afan, où l'on piège et que l'on abat pour les meilleurs champs, par opposition à la «friche» ekolok, milieu anthropique par excellence, qui entoure les villages comme un anneau. Ie village est une unité sociale séparée d'une autre par une zone de forêt vierge, nommée okang, «espace entre deux villages» («bosquet» en français local). Les Pygmées Kola distinguent aussi la "forêt» zii de la «friche" i'udi, et opposent leur propre hameau ndabo au village des essarteurs kw'clo, tout en distinguant ce hameau du camp forestier mbaa dépourvu de cultures vivrières.

Un point important concerne la mort et les défunts : le lieu où ils sont enterrés et l'attitude que l'on adopte vis-à-vis d'eux et de leurs âmes sont significatifs de l'attitude face à la nature. Ainsi pour less Pygmées Aka et Baka ‘[3]. les âmes des morts, redoutées, deviennent des forces immatérielles dans la forêt; dès après l'enterrement, qui a lieu dans le campement, le site est définitivement abandonné à la repousse de la forêt. Au contraire, chez les agriculteurs d'Afrique centrale, les défunts sont enterrés dans le village, souvent devant leur propre maison; les tombes font partie 
du paysage quotidien, au centre de la communauté, et hors de portée de la forêt. Dans ces sociétés, les cultes des ancêtres étaient fréquents dans un passé récent et les âmes des défunts sont toujours invoquées nommément pour le bien des vivants.

\section{L'espace est sacralisé}

Chaque religion traditionnelle possède un panthéon constitué de dieux créateurs du monde, d'esprits bénéfiques ou maléfiques, de héros démiurges, de monstres, et des âmes des défunts. Ces différents ensembles d'êtres surnaturels, qui peuplent le monde en même temps que les humains vivants, occupent différents endroits de l'espace (c'est le cosmos). Selon les peuples, ce cosmos prend différentes formes.

Pour les Wayampi de Guyane par exemple, l'univers est l'œuvre de plusieurs équipes d' architectes : il est constitué de quatre disques plats comme des grils à cuire le manioc, qui reposent les uns au-dessus des autres, soutenus par des pilotis géants. Un étage couvert de forêt est peuplé par les hommes, les démons et les ombres des morts. En dessous, un monde souterrain peuplé de paresseux géants où Soleil a ses abattis. Au-dessus, deux ciels : le premier est réservé au vautour, le second au Créateur et aux âmes des morts [cf. Grenand, 1982; Grenand \& Grenand, 1995].

D'une manière très globale, le ou les dieux créateurs vivent dans le ciel, alors que la forêt «vierge» est le domaine des esprits. Les rivières et les marécages abritent des esprits spécifiques. Pour chaque société, la disposition symbolique dans l'espace de ses êtres surnaturels permet de définir le sacré qui lui est propre, et en tout pre- mier lieu, d'opposer les aires spatiales sacrées aux aires relevant du domaine profane.

Ainsi, pour les Pygmées Aka de RCA, la forêt est le royaume des mânes; les zones hors de la forêt, c'est-à-dire la lisière, les villages (des essarteurs) et les rivières, sont hantées par de mauvais esprits étrangers - en ce sens, le village est un monde non humain, mauvais et dangereux; l'unique monde humain est le campement pygmée. Chaque sortie du campement pour aller en forêt est potentiellement dangereuse et doit être accompagnée de rituels protecteurs furtifs.

Chaque société possède des acteurs bumains liés aux faits de religion, des spécialistes (prêtres, chamanes, devins, guérisseurs...) qui ont, entre autres, des responsabilités particulières en regard de la forêt et des produits forestiers, animaux ou plus rarement plantes. En effet, si dans les sociétés de savane, l'agriculture donne lieu à des rites spécifiques, pour les sociétés des forêts tropicales, même chez les essarteurs, ce sont principalement les activités de chasse et de pêche qui sont accompagnées de rituels, organisés par des «ritualistes» spécialisés (même si l'acte de plantation est souvent ritualisé, comme en Amazonie).

En ce qui concerne la «géographie du sacré", c'est-à-dire l'organisation spatiale des activités religieuses, et à la différence des sociétés vivant en savane, il y a en forêt peu de sites sacrés, de lieux de culte ou de vénération. En particulier le phénomène des "bois sacrés", fréquent en Afrique de l'0uest, n'existe pas en forêt d'Afrique centrale ni en Amazonie. Par contre, on rencontre des lieux-dits porteurs d'histoire mythique, comme par exemple l'endroit où les oiseaux se parèrent de leurs couleurs, ou encore le fromager 
su pied duquel on accède au monde souterrain...

\section{Le fonctionnement de la nature fait l'obiet de représentations}

Toutes les sociétés forestières croient en un équilibre global entre les ressources de la nature, les forces surnaturelles et les hommes : les forces surnaturelles favorisent les activités des humains en leur procurant les ressources naturelles, animaux ou plantes. Toutes pensent que l'hamonie de la vie en société et une bonne communication avec les êtres surnaturels, grâce aux rituels et aux spécialistes, permettent l'efficacité des activités de production. En contrepartie, les hommes se donnent comme ligne de conduite de ne pas ahuser des ressources de la nature. Les maladies et la mort sont toujours attribuées aux forces surnaturelles et considérées comme des conséquences de la rupture des équilibres entre les hommes, les ressources naturelles et les esprits.

Ainsi, pour les Amérindiens Wayampi de Guyane, c'est un comportement pondéré vis-à-vis des animaux qui évitera aux hommes la maladie et le malheur : l'excès provoque un conflit avec les esprits maîtres des animaux. Pour les pêcheurs Ntomba du Zärre, les esprits des eaux s'adressent au pêcheur dans ses rêves et toute pêche excessive sera contrebalancée par le don d'une vie humaine [Pagezy \& Guagliardo, 1992].

\section{L'NOGGËEE, L'ETHNOLOUUE ET LA FAPẼ, FACE FU MONDE OCCIDENTAL}

l'ethnologue que je suis commença sa vie de jeune chercheur en s'installant dans un campement au fond de la forêt, loin des routes, pour comprendre l'intimité éternelle d'un groupe humain avec un écosystème remarquable. II fallait être bien naif pour oublier que les routes s'allongent et que des endroits inaccessibles cessent de l'être à tout instant de la marche des siècles. (Qu'il le veuille ou non, l'ethnologue, à mesure qu'il cesse d'être jeune, rencontre sur son chemin au bout de la forêt les effets de son propre monde, ce que l'on nomme curieusement "progrès", "modernisme» ou encore "développement». Il observe l'érosion d'un système qu'il a connu et reste perplexe sur les capacités de sursaut des habitants de son vieux campement. Il se croit porteur d'un savoir, voire d'un message et enrage que son avis ne soit jamais sollicité.

Lorsqu'enfin il sera consulté, l'ethnologue des forêts prendra conscience de l'ampleur du chantier qui s'ouvre devant lui; il devra mesurer toute la distance qui sépare son laboratoire des bureaux des «hommes d'actions»; il lui faudra aussi comprendre un langage et des concepts nouveaux pour lui. Les questions se presseront alors à son esprit et il devra définir sa place dans ce monde nouveau.

J'ai passé près de vingt ans à étudier et à décrire le mode de vie et l'ethnoécologie d'une société de chasseurs-cueilleurs de la forêt d'Afrique centrale, puis à tenter d'en dégager l'histoire, à travers la langue et la culture ${ }^{\left[{ }^{[j]}\right.}$. Alors que ce travail approche de son terme, les conditions locales ont bien changé, au point de rendre le groupe étudié presque méconnaissable. Dans le même temps, les actions se multiplient à l'égard d'une région préalablement laissée à elle-même. L'ethnologue peut-il dès lors rester en dehors du 
processus, et dans le cas contraire, quelle peut être son action?

Cependant, avant d'aller plus avant, il n'est pas sans intérêt de s'arrêter un instant sur deux notions nouvelles, qui viennent de faire irruption dans le champ conceptuel des chercheurs, devenant «incontournables» : la biodiversité et le développement durable.

\section{Biodiversité et développement durable}

À la suite du Sommet de la Terre tenu à Rio en juin 1992 (CNUED), une Convention internationale sur le biodiversité entre en vigueur le 29 décembre 1993. Celle-ci pose le principe d'une réglementation de l'appropriation par l'homme des ressources vivantes, de la protection de la diversité biologique et de l'utilisation durable de ses composantes. C'est ainsi instaurer officiellement la nécessité d'une gestion durable des écosystèmes. De plus, cette convention reconnaît également le rôle des «populations indigènes»:

- «De nombreuses communautés indigènes et locales dépendent étroitement des ressources biologiques. Les gouvernements devraient préserver les méthodes traditionnelles de conservation et d'utilisation durable de la diversité biologique et s'en inspirer aussi largement que possible, en associant pleinement les populations concernées à leurs efforts.» [Sachs, 1993: 100-102].

De nombreux organismes internationaux se préoccupent maintenant des populations locales, et de leur rôle dans la "gestion durable», Banque Mondiale [cf. Goodland, 1982] en premier lieu. mais aussi par exemple l'Organisation internationale des bois tropicaux (OIBT / ITTO) qui mentionne les communautés locales dans ses cri- tères de mesure de l'aménagement durable des forêts tropicales, ou encore le Bureau international du travail de Genève.

Dans ce nouveau contexte, quelques organismes politiques s'interrogent sur «les populations indigènes" et la place à leur réserver dans le cadre de leurs actions. Quelques-uns se tournent vers les chercheurs en sciences humaines: «vous nous dites qu'il faut tenir compte de « vos gens», soit : de quoi ont-ils besoin pour vivre?». Et l'anthropologue est piégé: il ne sait pas répondre à cette question. Les connaissances de base sur l'écologie, l'économie et l'alimentation de la majorité des populations forestières sont trop fragmentaires pour livrer une image lisible. Pour de nombreuses ethnies, on ne connaît toujours pas les surfaces employées pour l'agriculture, pour la chasse, la cueillette et la pêche; on ne connaît ni les cycles des rotations agricoles, ni les régimes alimentaires ni leur efficacité.

Il faut bien reconnaitre que la belle période de l'écologie dans les sciences humaines n'a pas eu les suites que l'on attendait; la désaffection des étudiants et des chercheurs est patente et les recherches n'ont que peu été conduites, au cours des vingt dernières années. Face aux questions des praticiens et des applicateurs, les publications des anthropologues paraissent bien ésotériques. Ie décalage entre les débats des chercheurs et les questions urgentes, très pratiques, liées à l'évolution des conditions de vie modernes apparaît insurmontable.

\section{Qu'est-ce que la biodiversité ?}

Que cache ce terme, abréviation de "diversité biologique»? 
- "Biological diversity" encompasses all species of plants, animals, and microorganisms and the ecosystems and ecological processes of which they are parts. It is an umbrella term for the degree of nature's diversity, including both the number and frequency of ecosystems, species and genes in a given assemblage. It is usually considered at three different levels: genetic diversity, species diversity, and ecosystem diversity." [McNeely \& al., 1990 : 17].

Selon la définition donnée par l'un des créateurs de ce concept, E.0. Wilson:

- «Biodiversité: la diversité des organismes considérée à tous les niveaux, depuis les variants génétiques appartenant à la même espèce jusqu'aux gammes des espèces et aux gammes des genres, familles, et des catégories taxinomiques de plus haut niveau. Elle comprend également la diversité des écosystèmes, lesquels sont constitués à la fois de la communauté des organismes vivant au sein d'habitats particuliers et de l'ensemble des conditions physiques qui y règnent.» [Wilson, 1993 : 454].

Plus simplement, cette diversité biologique, n'est-ce pas ce que naguère d'aucuns appelaient «la nature»?

La naissance de ce concept est directement liée à l'accélération des destructions environnementales et à la disparition croissante d'espèces vivantes.

«Il est clair que nous sommes en plein cœur de l'une des grandes crises d'extinctions de l'histoire de la Terre.» [Wilson, op.cit. : 328].

Cette constatation fonde une conviction profonde : "... l'humanité a déclenché la sixième grande crise d'extinction, précipitant dans le néant une grande proportion des espèces qui nous accompagnent, et ce en l'espace d'une génération.» [Wilson, op.cit. : 46].

D'où il découle, par un raisonnement drastiquement simpliste, que l'espèce humaine est intrinsèquement destructrice : «Les chasseurs humains ne font du bien à aucune espèce. C'est là une vérité générale et la clé de toute la triste histoire.» [Wilson, op.cit. : 296]; «I)e la préhistoire à aujourd'hui, les cavaliers de l'apocalypse écologique se sont illustrés dans la chasse effrénée, la destruction des habitats, l'introduction d'animaux tels que rats ou chèvres, et les maladies transportées par ces derniers animaux.» [Wilson, op.cit. : 297].

Dès lors, le naturaliste est porté par un rêve, une quête peut-être illusoire : "Comment était la Terre dans son état naturel, avant l'homme ? C'est le mystère que nous avons reçu pour mission de résoudre, ce qui devrait nous permettre de remonter au lieu de naissance de notre esprit.» [Wilson, op.cit. : 404].

L'ethnologue ne peut manquer d'être heurté par une telle vision des choses, une lecture de l'histoire de l'humanité aussi abrupte : en posant que l'homme est mauvais par essence, on amalgame (consciemment ?) toutes les sociétés et leurs actions, destructrices ou non, ce qui est un artifice très commode pour éviter de regarder en face les responsabilités de notre société...

\section{De l'utilité de la nature}

On peut cependant constater que la «biodiversité» recouvre tout à la fois une notion, celle d'ensemble des espèces vivantes " the total of genes, species, and ecosystems on earth", 
[McNeely \& al., 1990 : 11], mais aussi un message d'actions de préservation. En effet, il est reconnu que les destructions sont liées, d'une part, à la surexploitation forestière, d'autre part, à l'accroissement démographique. Dès lors, un second concept est devenu inséparable du premier, celui de «développement durable» (sustainable).

«Promotion d'un développement viable. Les paysans pauvres du 'Tiers Monde sont prisonniers d'une spirale descendante liant l'accroissement de la pauvreté à une destruction accrue de la diversité. [...] Le problème se ramène alors à cette question : comment les habitants des pays en développement pourront-ils tirer un revenu décent de la terre sans détruire celle-ci ?" [Wilson, 1993: 377-78].

En fait apparait une double question: comment protéger la biodiversité contre les populations rurales, mais aussi comment la protéger contre les entreprises d'exploitations en provenance du Nord? C'est là que se met en place un raisonnement utilitariste. La nature est riche en espèces interdépendantes, toutes nécessaires à son fonctionnement (même si les mécanismes peuvent nous être encore inconnus); l'homme est destructeur mais néanmoins il dépend de certaines autres espèces pour sa survie; indéniablement il ne connaît et n' utilise qu'une faible partie des espèces naturelles et découvre chaque jour de nouveaux produits utiles : comme on n'a encore aucune idée des ressources potentielles qui se cachent dans les millions d'espèces vivantes, il est nécessaire de conserver la biodiversité pour garantir aux générations futures l'accès aux ressources qu'elles découvriront.

\section{Il s'agit donc de justifier la préservation de} la nature par son utilité potentielle. Ainsi, la biodiversité apparaît-elle comme un concept mis au point par les naturalistes dans une pathétique tentative pour justifier l'existence même de la nature sauvage contre les prétentions de l'Homme dans son ultime effort pour conquérir et asservir la totalité du globe...

Mais l'ethnologue relèvera qu'il ne s'agit pas, malgré les apparences, de n'importe quel homme, ni de l'espèce humaine dans son ensemble. Ces entreprises d'exploitations sont celles de la civilisation des hommes du Nord, selon l'expression admise de nos jours, c'est-à-dire l'économie des Occidentaux, celle du fameux $\mathrm{G} 7$ des «nations les plus riches du monde ", relayée par les nations imitatrices. La mondialisation de cette économie capitaliste conduit à utiliser la même logique marchande dans l'argumentation pour défendre la nature, pour contrer ces entreprises. N'est-ce pas une fois encore l'arrogance des Blancs qui est en jeu, avec l'expansion de leur modèle de société et d'économie?

Aussi généreuse soit-elle, même l'argumentation utilitariste sur les ressources potentielles ne laisse pas d'être ambiguë. Des ressources nouvelles, mais nouvelles pour qui, et à l'usage de qui? Ne sont-elles pas nouvelles pour les gens du Nord, mais nullement pour les habitants ancestraux de ces régions équatoriales et ne seront-elles pas utiles principalement pour les marchés du Nord?

«L.a plupart des consommateurs du Nord n'ont pas encore savouré l'orange de Quito, le génipape, le ramboutan et les quasi légendaires durion et mangoustan, regardés par les connais- 
seurs comme les fruits numéros un du monde entier.» [Wilson, 1993: 337].

\section{Indigènes el forêts}

On a vu que les habitants des zones naturelles sont inclus dans la notion de biodiversité, certes pas en tant qu'espèces vivantes, mais en tant qu'agents. Indéniablement il existe une relation directe entre futur de la forêt équatoriale et devenir des peuples indigènes. Ces populations (qu'on les nomme traditionnelles, indigènes, ou de tout autre nom, importe peu) sont concernées à deux niveaux par les politiques liées à la biodiversité : conservation et gestion. Ce que l'on peut résumer par les questions suivantes: dans quelle mesure la préservation de l'écosystème forestier serait-elle contrariée par la présence des sociétés forestières indigènes? le développement économique des forêts constitue-t-il un bénéfice ou au contraire une menace pour les habitants des régions forestières?

\section{A - la conservation}

Lorsque des processus de conservation sont mis en place, n' importe quelle population rurale naguère isolée peut se retrouver en plein coeur d'une zone protégée; elle se trouve de ce fait mise en demeure de démontrer que son style de vie immémoriale est «compatible avec le maintien de la biodiversité». Nous revenons là à la question fondamentale: les populations traditionnelles détruisent-elles leur environnement? Pour préserver "la biodiversité ", faut-il chasser les indigènes? En d'autres temnes, les indigènes sontils nécessairement cause de déforestation ou d'appauvrissement faunistique?
Il est de bon ton d'accuser l'agriculture itinérante de faire partir en fumée ces beaux arbres, source de richesses évaporées. . Mais accuser les essarteurs d'être les destructeurs de la forêt, c'est faire un amalgame inique entre d'un côté ces hommes, vivant dans les forêts depuis plusieurs millénaires, et dont l'agriculture ne constitue qu'un volet des activités de production, associée à la chasse, la pêche et la cueillette, et de l'autre côté les immigrants. Ces derniers peuvent venir d'horizons très diversifiés : ce peuvent être de petits paysans issus d'autres régions agricoles déjà en crise; ce peuvent être aussi des prolétaires sans compétence agricole particulière, embauchés par de grands patrons ou de grandes entreprises. Ces immigrants ne pratiquent pas lagriculture itinérante sur brûlis, mais le défricbage par le feu : leur but est la conquête définitive de l'espace pour la culture ou l'élevage. Leur type d'exploitation est ensuite permanent, sans abandon des cultures, jachère ni rotation agricole. ('est la différence majeure entre ces deux types d agriculture. Et s'il y a une déforestation dont le feu de paysans serait la cause, c'est bien aux immigrants qui pratiquent une agriculture permanente qu'il faut l'attribuer. Il faut aussi rappeler que le système de l'agriculture itinérante est aulorégénérant; au contraire, quand on passe à une agriculture permanente, il y a nécessité d'apports nutritifs, d'intrants, pour régénérer le sol.

En réalité, la déforestation n'est pas liée aux activités des populations traditionnelles forestières. Elle est liée principalement à la nécessité pour les États de la ceinture intertropicale de trouver des ressources monétaires, en particulier grâce aux produits d'exportation. Elle est liée à une 
politique très consciemment pratiquée par les gouvernements de pousser vers la forêt des milliers de prolétaires afin de diminuer la pression qui pèse sur les centres urbains. Elle est liée encore au commerce international, aux besoins de nos contrées à nous; ces produits d'exportation que les États équatoriaux recherchent, c'est d'abord le bois, mais aussi les minerais, l'énergie, en particulier en Asie où nombre de pays construisent des barrages pour exporter de l'électricité, noyant de ce fait leurs forêts. Il y a aussi l'agriculture industrielle du café, hévéa, cacao, poivre, palmier à huile, etc. et enfin, l'élevage extensif pour la production de viande. Tous ces biens sont destinés à la production de devises pour les ÉtatsNations, rien de cela ne constitue une ressource qui retournerait aux populations préexistant dans la zone où ces activités se développent. Qui détruit la forêt?

\section{B - la gestion}

C'est précisément l'échec des politiques de culture de rente (café, cacao, etc.) ou d'élevage extensif qui conduit certains gouvernements des pays tropicaux à rechercher d'autres activités; ils disposent pour cela d'un nouvel outil pour imposer leur loj économique à toutes les populations rurales : l'extructivisme, c'est-à-dire la collecte commerciale de produits sauvages.

Il est certes satisfaisant pour l'esprit de remplacer l'exploitation forestière ou la culture du café par la collecte de plantes sauvages, dont la vente assurerait aux habitants des forêts un revenu utile. Toutefois, la distance est grande entre le noyer du Para perdu dans la forêt amazonienne et l'épicerie fine de Paris ou de New
York. Comment garantir que l'Indien gagnera bien son labeur, sans enrichir une demi-douzaine d'intermédiaires plus calculateurs que lui, voire sans être asservi par eux? Si l'on songe qu'un pays comme la France, doté de lois et de règlements efficaces, et appliqués, est incapable de laisser vivre de leur travail ses derniers pêcheurs artisanaux (car ceux-là aussi vivent de la biodiversité), on reste dubitatif sur les possibilités de justice de certains pays du 'liers Monde...

Comment garantir aussi que le récolteur restera sage et que l'attrait du gain ne le poussera pas à une exploitation dévastatrice des ressources sauvages? N' avons-nous pas détruit par la collecte le sylphium parfumé des Anciens ou le bois-brésil emblématique? N'est-on pas en passe de détruire les peuplements de rotin de Malaisie ou de Boméo? Comment développer l'extractivisme des produits végétaux, mais interdire le prélèvement à but commercial des produits animaux c'est-à-dire le braconnage?

Cela sans parler du problème philosophique que pose le fait de vouloir rendre commerçants et producteurs des groupes qui ne le sont pas...

\section{Differences d'attitudes}

L'une des difficultés auxquelles on se trouve confronté en terme d'amélioration des relations entre l'Homme et le milieu, est l'incapacité du développeur appartenant aux sociétés modernes industrialisées à pénétrer les aspects non matériels, la philosophie de la vie de la population impliquée. Quelle que soit la bonne volonté, on se rend coupable de socio-centrisme et l'on tend à projeter dans l'esprit d' individus étrangers à notre culture des conceptions qui n'ont de valeur opé- 
ratoire que dans la nôtre. C'est ainsi que "l'optimal foraging theory » est profondément influencée par des conceptions d'efficacité matérielle qui ont cours dans la société américaine compétitive et agressive d'aujourd'hui plutôt que dans les sociétés traditionnelles. La recherche crue de l'efficacité technique est étrangère à la réalité indigène.

Les sociétés traditionnelles sont gouvernées par des pensées non cartésiennes. Nombre d'êtres humains, y compris quelques Occidentaux, ne croient pas totalement que le Cosmos puisse être expliqué rationnellement ni qu'il puisse être contrôlé matériellement. Àl'extérieur de la civilisation occidentale pour laquelle l'environnement apparaît comme un objet matériel dénaturé et profane que l'on peut rudement exploiter sans crainte, la plupart des sociétés traditionnelles possèdent une sorte de concept panthéique de la nature, pensée et vécue comme une entité vivante, peuplée aussi bien d'êtres surnaturels que d'êtres vivants avec lesquels les hommes entretiennent des relations de type varié [cf. de Garine, 1991].

C'est pourquoi il importe de mettre en évidence les représentations symboliques et religieuses que se font les populations indigènes de la forêt dans laquelle elles vivent, c'est-à-dire les valeurs culturelles qu'elles lui prêtent et la place qu'elles se donnent en son sein. Le développeur et le conservateur de la nature ne pourront pas s'appuyer sur une spatialisation délimitée de lieux traditionnellement protégés par les membres d'une société forestière. Par contre, ils seront confrontés à des philosopbies qui fondent la coexistence de la société humaine et des ressources du milieu naturel. Ils devront en tenir compte dans leur politique : la conscience des territoires naturels est forte chez les peuples forestiers et leur cosmogonie est basée sur l'usage modéré et le contrôle de l'accès aux ressources sauvages; vouloir les en séparer ne peut rencontrer la moindre compréhension.

\section{Conclusion}

l.a relation homme/nature s'inscrit dans une notion plus large d'équilibre global entre santé/maladie et bonheur/malheur. C'est le maintien de cet équilibre qui sera le meilleur appui pour la politique de conservation. On constate en effet que les excès de prélèvement apparaissent en situation de transition, de basculement et de rupture dans les sociétés traditionnelles. Les usages ne restent mesurés que dans un contexte d'équilibre social et culturel. Les excès indéniables observables par exemple en Afrique Centrale ou en Amazonie doivent être lus dans leur globalité : ils s'exercent dans un contexte de crise morale (appauvrissement des religions traditionnelles), de crise économique (monétarisation et ouverture commerciale) et d'augmentation du potentiel technique (armes à feu, filets de nylon). L'ensemble mène l'individu, chasseur ou pêcheur, à dépasser des seuils naguère fixés au seul approvisionnement de sa maisonnée, pour atteindre des taux de "production» intéressant le marché régional (commerce de viande ou de poisson fumé) et financièrement rentables (dans un premier temps tout du moins). Les acteurs de la conservation de la nature devront donc tenir compte de l'analyse de l'ensemble de ces phénomènes avant d'élaborer leurs politiques. 
Les populations indigènes des forêts denses humides, répétons-le, sont pour l'immense majorité, des essarteurs, pratiquant l'agriculture itinérante sur brûlis. Elles sont nombreuses et ne forment pas des «minorités» : près de 12 millions de personnes vivent traditionnellement de la forêt équatoriale, elles constituent l'essentiel des forces rurales de ces forêts, elles y vivent depuis longtemps et n'en sont pas destructrices. N'ont-elles pas conservé la biodiversité jusqu'à l'aube du XXe siècle? L'avenir des populations indigènes est très directement lié à l'avenir des forêts : c'est une gestion durable des forêts équatoriales qui assurera aussi un futur aux populations indigènes. Mais l'inverse est tout aussi urai : les forêts équatoriales n'ont d'avenir que dans le maintien en leur sein de populations qui savent traditionnellement les exploiter sans les détruire.

Les populations forestières ont très tôt participé à l'exploitation des ressources forestières pour des régions extérieures, soit par des réseaux locaux de commerce, soit pour des réseaux internationaux. Il n'y a donc aucune raison pour actuellement les laisser en dehors des décisions et des activités concernant les forêts - elles peuvent être beaucoup plus que des sources de manœuvre. À fortiori si l'on cherche à mettre en place des techniques de développement durable : faire participer les paysans et les écouter c'est profiter de savoirs ancestraux qui manquent cruellement aux Occidentaux, c'est aussi profiter de l'entbousiasme de populations qui se sentent concernées par leur futur.

Enfin, il faut insister sur le fait que les luttes actuelles menées par certains groupes forestiers de par le monde ne visent pas à préserver un genre de vie unique, mais à garantir à leurs générations futures un espace minimal et des valeurs culturelles dans lesqueis ils se reconnaissent; il s'agit surtout de gagner le droit à décider soi-même des choix à opérer pour le futur.

À cela, l'ethnologue, pas plus que l'agent de développement, ne peuvent rester indifférents. 


\section{AEMERCIEMENTS}

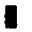

Ce texte doit beaucoup aux

discussions et réflexions du groupe de travail que nous avons constitué lors de la réalisation du rapport Situation des populations indigènes des forêts denses el bumides, Françons: A!randl, Françols: Grinand, Pilirk: Grinanj) et DAOU JoIRIS, ainsi qu'aux commentaires d'loor [Be Garinf.

\section{NOTES}

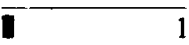

Rappelons la première définition du Petit Roberl, par exemple:

«indigène: qui est né dans le pays dont il est question".

- 2

On trouvera plus de détail dans le rapport remis à la Commission

Européenne (DG XI),

BAHICHET \& It: MnReT (sous la direction de), 1994, Situation des popinlations indigènes des forêts dersses et bumides.

\section{3}

On trouvera des exemples de régintes alimentaires forestiers dans Se nourrir en forêt équatoriale (HIsink \& As.. 1989) et surtout dans de nombreux chapitres de l'ouvrage Iropical forests. people and food (HiAjlK \& Al.. 1993).

\section{$\overline{4}-\overline{4}$}

On se reportera au rapport Elude comparative de la représentation symbolique des arbres et de la forêt équaloriale. En 1995 pour la Commission européenne (I)G XI).

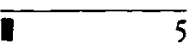

Pour les Aka, cf. BAinsitht, 1985, pour les Baka cf. Jolk1s, 1993a, 1993b, 1996.

[ 6

BaHUCHT, 1985, 1992, Thomas \& BNHLCHET, 1981 